\title{
Metadata and Table Caption Correction: What Do Patients Say About Doctors Online? A Systematic Review of Studies on Patient Online Reviews
}

\author{
Y Alicia Hong ${ }^{1,2}$, PhD; Chen Liang ${ }^{3}$, PhD; Tiffany A Radcliff ${ }^{2}, \mathrm{PhD}$; Lisa T Wigfall ${ }^{4}, \mathrm{PhD}$; Richard L Street ${ }^{5}, \mathrm{PhD}$
}

${ }^{1}$ Department of Health Administration and Policy, George Mason University, Fairfax, VA, United States

${ }^{2}$ School of Public Health, Texas A\&M University, College Station, TX, United States

${ }^{3}$ Arnold School of Public Health, University of South Carolina, Columbia, SC, United States

${ }^{4}$ Department of Health Kinesiology, Texas A\&M University, College Station, TX, United States

${ }^{5}$ Department of Communication, Texas A\&M University, College Station, TX, United States

\section{Corresponding Author:}

Y Alicia Hong, $\mathrm{PhD}$

Department of Health Administration and Policy

George Mason University

4400 University Drive, MS 1J3

Fairfax, VA, 22030

United States

Phone: 17039931929

Email: yhong22@gmu.edu

\section{Related Article:}

Correction of: http://www.jmir.org/2019/4/e12521/

(J Med Internet Res 2019;21(7):e14823) doi: 10.2196/14823

The authors of "What Do Patients Say About Doctors Online? A Systematic Review of Studies on Patient Online Reviews" (J Med Internet Res 2019;21(4):e12521) made an error in the caption of Table 2. It previously read "Summaries of published studies on patient online reviews (63 studies consisting of 69 articles)" but has now been changed to "Studies that compare patient online reviews with traditional healthcare quality indicators".

The lead author, Y Alicia Hong, now has an additional affiliation (Department of Health Administration and Policy, George Mason University, Fairfax, VA, United States) in addition to her previous affiliation (School of Public Health, Texas A\&M University, College Station, TX, United States). This has bumped the numbering of all other affiliations by one, although affiliations remain the same for all other authors.

Y Alicia Hong is also the corresponding author and wishes to use her new affiliation's information for correspondence. The previous contact information was as follows:

\section{$Y$ Alicia Hong, PhD \\ School of Public Health \\ Texas A\&M University}

\section{Adriance Lab Road \\ College Station, TX, 77843-1266 \\ United States}

Phone: 19794369343

Email: yhong@sph.tamhsc.edu

The new contact information is as follows:

$Y$ Alicia Hong, PhD

Department of Health Administration and Policy

George Mason University 4400 University Drive, MS

$1 J 3$

Fairfax, VA, 22030

United States

Phone: 17039931929

Email: yhong22@gmu.edu

The correction will appear in the online version of the paper on the JMIR website on July 18, 2019, together with the publication of this correction notice. Because this was made after submission to PubMed, PubMed Central, and other full-text repositories, the corrected article also has been resubmitted to those repositories. 
This is a non-peer-reviewed article. Submitted 26.05.19; accepted 13.06.19; published 18.07.19.

Please cite as:

Hong YA, Liang C, Radcliff TA, Wigfall LT, Street RL

Metadata and Table Caption Correction: What Do Patients Say About Doctors Online? A Systematic Review of Studies on Patient Online Reviews

J Med Internet Res 2019;21(7):e14823

URL: http://www.jmir.org/2019/7/e14823/

doi: $10.2196 / 14823$

PMID: $\underline{31322126}$

(CY Alicia Hong, Chen Liang, Tiffany A Radcliff, Lisa T Wigfall, Richard L Street. Originally published in the Journal of Medical Internet Research (http://www.jmir.org), 18.07.2019. This is an open-access article distributed under the terms of the Creative Commons Attribution License (https://creativecommons.org/licenses/by/4.0/), which permits unrestricted use, distribution, and reproduction in any medium, provided the original work, first published in the Journal of Medical Internet Research, is properly cited. The complete bibliographic information, a link to the original publication on http://www.jmir.org/, as well as this copyright and license information must be included. 